\section{Idiopathische Lungen- fibrose: Wie verlässlich sind Informationen aus dem Internet?}

Fisher JH et al. Accuracy and Reliability of Internet Resources for Information on Idiopathic Pulmonary Fibrosis. Am J Respir Crit Care Med 2016; 194: $218-225$

Viele Patienten nutzen das Internet als Quelle für Gesundheitsinformationen: Rund 4,5\% aller Internetsuchen betreffen Gesundheitsthemen. Doch wie verlässlich sind diese Informationen? J. H. Fisher et al. überprüften dies nun für die idiopathische Lungenfibrose.

Die Autoren suchten hierfür mit Hilfe der drei gebräuchlichsten Internet-Suchmaschinen Google, Yahoo und Bing nach schriftlichen Online-Informationen zur idiopathischen Lungenfibrose. Die 200 Top-Treffer jeder Suchmaschine überprüften sie auf ihre Eignung. Ausgeschlossen waren Websites, die eine Registrierung erforderten oder Gebühren verlangten sowie professionelle Seiten für Fachleute im Gesundheitswesen. Die gefundenen Seiten wurden von den Autoren unter anderem bezüglich des Ursprungs der Informationen, des Publikationsdatums und der Lesbarkeit beurteilt. Die Qualität wurde mithilfe des validierten Instruments „DISCERN“ sowie Kriterien des Journal oft the American Medical Association bewertet.

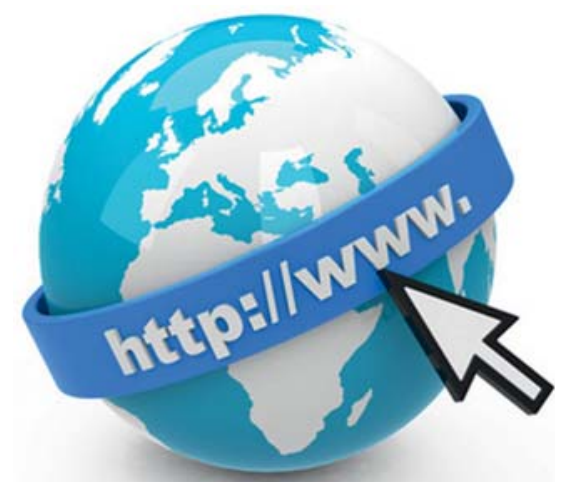

Das Internet bietet jedem schnell zugängliche Informationen zur idiopathischen Lungenfibrose. Aber wie gut ist „Doktor Internet“ wirklich?

Bildquelle: beermedia/www.Fotolia.com

Die Analyse der ersten 200 Treffer jeder der Suchmaschinen ergab 350 eigenständige Websites, von denen 181 die Einschlusskriterien erfüllten; Yahoo verzeichnete dabei die meisten Seiten (118 vs. jeweils 90 bei Google und Bing). Informationen aus wissenschaftlichen Quellen wie akademischen Institutionen zeigten hierbei mehr Gehalt als Seiten mit persönlichen Kommentaren wie beispielsweise Blogs. Jedoch fanden sich auf den meisten Seiten unvollständige oder ungenaue Informationen. Nicht indizierte oder gar schädliche medikamentöse Therapien der idiopathischen Lungenfibrose wurden auf $48 \%$ der Websites als potenzielle Behandlungen beschrieben und am häufigsten auf Seiten von Stiftungen oder Interessensverbänden empfohlen. Azathioprin und Corticoste- roide wurden auf 13,3\% bzw. 30,6\% derjenigen 98 Webseiten als potenzielle Therapie diskutiert, die nach der Publikation von Daten aktualisiert worden waren, die eine Schädlichkeit dieser Medikamente belegte. Die Qualität der Information war auf allen Arten von Seiten dürftig, aber auf Nachrichten- bzw. Medienseiten und Blogs schlechter als auf Seiten wissenschaftlicher Organisationen und Interessensverbände.

FAZIT

An Patienten gerichtete Online-Informationen zur idiopathischen Lungenfibrose sind häufig unvollständig, ungenau und überholt. Es gibt keine verlässliche Methode für Patienten um Seiten zu identifizieren, die adäquate Informationen liefern, so die Autoren.

Dr. med. Johannes Weiß, Bad Kissingen 